\title{
Mass transfer effects on linear wave propagation in diluted bubbly liquids
}

\author{
D. Fuster ${ }^{1}$, F. Montel ${ }^{2}$ \\ ${ }^{1}$ Sorbonne Universités, UPMC Universit Paris 06, CNRS UMR 7190, Institut Jean Le Rond \\ d'Alembert, F-75005 Paris, France \\ 2 TOTAL SA, CSTJF, Avenue Larribau, 64018 Pau, France.
}

(Received 2015)

This article investigates the importance of mass transfer effects in the effective acoustic properties of diluted bubbly liquids. The classical theory for wave propagation in bubbly liquids for pure gas bubbles is extended to capture the influence of mass transfer on the effective phase speed and attenuation of the system. The vaporization flux is shown to be important for systems close to saturation conditions and low frequencies. We derive a general expression for the transfer function that relates bubble radius and pressure changes solving the linear version of the conservation equations inside, outside and at the bubble interface. Simplified expressions for various limiting situations are derived in order to get further insight about the validity of common assumptions typically applied in bubble dynamic models. The relevance of the vapor content, the mass transfer flux across the interface and the effect of the bubble interface temperature variations is discussed in terms of characteristic nondimensional numbers. Finally we derive the various conditions that must be satisfied in order to reach the low frequency limit solutions where the phase speed does no longer depend on the forcing frequency.

\section{Introduction}

The effect of bubbles on the process of wave propagation in liquids is an open scientific problem with many applications fields such as ultrasonic fluid flow monitoring and geophysics (Kuster \& Toksöz 1974; Lynnworth 2013). One family of models typically used for wave propagation in bubbly flows applies for dilute systems. Most of these models (Van Wijngaarden 1968; Chapman \& Plesset 1971; Prosperetti 1977; Sangani 1991; Zhang \& Prosperetti 1997; Ando et al. 2009) succeed reproducing experimental results for frequencies below resonance in situations where the amount of vapor is negligible (Silberman 1957; Cheyne et al. 1995; Wilson et al. 2005; Leroy et al. 2008). These theories have been recently improved at frequencies above the bubble natural frequency. For instance, high frequency corrections have been proposed by Ando et al. (2009), while Fuster et al. (2014) propose correction terms in order to capture direct bubble-bubble interactions based on the results obtained from a full non-linear model (Fuster \& Colonius 2011).

Theoretical models for linear wave propagation in bubbly liquids require to model the response of a single bubble to an external pressure excitation. This response is obtained through the linearization of the Rayleigh-Plesset equation and the solution of the mass, momentum and energy conservation equations inside the bubble. Expressing the amplitude of the bubble radius oscillation as a function of the external pressure amplitude allows us to define a bubble resonance frequency and damping factor, see Ainslie \& Leighton (2011) for a review. These parameters depend on the so-called transfer function, which relates bubble pressure and volume changes in the linear regime. Prosperetti et al. 
(1988) propose an expression capturing the heat transfer exchange between the bubble and its environment assuming that the bubble interface temperature is constant and neglecting mass transfer effects across the interface. This function can be used to define an effective state equation for the bubble interior of the type $p_{b} V_{b}^{\gamma_{\text {eff }}}=C$, where $p_{b}$ is the bubble pressure, $V_{b}$ is the bubble volume and $\gamma_{\mathrm{eff}}$ is a frequency dependent effective coefficient that tends to recover the isothermal limit for low frequencies $\left(\gamma_{\text {eff }}=1\right)$ and the adiabatic response for very large frequencies, $\gamma_{\text {eff }}=\gamma$, where $\gamma$ is the gas polytropic coefficient.

Wave propagation in systems where mass transfer effects become relevant have been mainly investigated for large void fractions far from the diluted limit (Mecredy \& Hamilton 1972; Kieffer 1977; Ardron \& Duffey 1978; Landau \& Lifshitz 1987). For low enough frequencies, Kieffer (1977) and Landau \& Lifshitz (1987) derive limiting expressions assuming that the far field pressure and temperature are related through the saturation conditions. Models accounting for transient heat, mass and momentum transport have been proposed by Mecredy \& Hamilton (1972), Ardron \& Duffey (1978) and more recently by Saurel et al. (2008).

In diluted systems, mass transfer is expected to influence the local bubble response without significantly influencing the far field temperature as the liquid plays the role of an infinite energy reservoir for the bubble oscillation. In this case, one needs to derive corrected expressions to relate bubble radius and external pressure changes. Hao \& Prosperetti (1999) consider the problem of mass transfer in pure vapor bubbles by assuming saturation inside the bubble at every instant. Preston et al. (2007) solve the linearized problem of the dynamics of air/vapor bubbles for situations where transient vaporization effects and the heat transport in the liquid boundary layer is not a controlling mechanism. Prosperetti \& Hao (2002) also discuss interesting phenomena induced by mass transfer on the dynamics of bubbles and Prosperetti (2015) presents a simplified model for the influence of mass transfer on the sound-speed of a gas-vapor bubbly liquid that reveals that mass transfer effects play an important role of the propagation of waves at low frequencies.

The process of wave propagation near saturation conditions for diluted systems is less investigated experimentally. Coste et al. (1990) report evidence of a strong decrease of the sound velocity at low void fractions when approaching the saturation curve in diethylether. Unfortunatelly, the study mainly reports data for large void fractions, where the diluted limit conditions are not met.

In this work we extend the classical linear theory for disperse bubbly liquids to include mass transfer effects across the interface and the diffusion of heat in the liquid. The goals of this study are twofold. First, we want to evaluate the influence of the mass transfer process in the bubble dynamic response and to discuss typical assumptions used in the literature. Second, we want to quantify the importance of mass transfer effects on the effective acoustic properties of the medium. To that end, the full model that accounts for transient mass transfer effects is presented in Section 2. In Section 3 we present the linearization procedure followed to derive the expressions for the bubble resonance frequency, damping factor, mass transfer flux, interface temperature variations and the effective sound phase speed and attenuation of the gas-vapor-liquid mixture (in the following we will simply name them phase speed and attenuation). In Section 4 we present a summary of the model. Section 5 presents some numerical results to gain further 
insight about mass transfer effects in the process of wave propagation, discussing the relevance of various mechanisms on the bubble response as a function of the vapor content and frequency. Section 6 derives low frequency limiting solutions and the conclusions are presented in Section 7.

\section{Full model}

The linearized equations for wave propagation in bubbly media can be derived from basic principles using the conservation equations applied to the averaged mixture. Typically the separation of scales assumption is used to write the averaged equations for the large scale wave propagation problem. Thus, neglecting the influence of liquid viscosity at large scales, we write the mass and momentum conservation equations as

$$
\begin{gathered}
\frac{1}{\rho c^{2}} \frac{D p}{D t}+\nabla \cdot v=\frac{\partial \beta}{\partial t}, \\
\rho \frac{D v}{D t}=-\nabla p,
\end{gathered}
$$

where $t$ is the time coordinate, $v$ is the fluid velocity, $p$ is the pressure and $\rho$ is the average density defined in terms of the void fraction $\beta$, the liquid's density, $\rho_{l}$, and bubble's density $\rho_{b}$ as $\rho=(1-\beta) \rho_{l}+\beta \rho_{b}$. The void fraction $\beta$ is defined using a probabilistic function for the bubble equilibrium radius, $f(a)$, and the number of bubbles per unit volume, $n$,

$$
\beta=\frac{4}{3} \pi n \int_{0}^{\infty} a^{3} f(a) d a .
$$

The system of equations above requires to derive an equation to relate external pressure changes with the local bubble radius variation. This equation is found by solving the conservation equations inside and outside the bubble at the local scale. If we impose spherical symmetry for the bubble oscillation, the basic equations for a system of $N$ species can be written as

$$
\begin{aligned}
\frac{D \rho}{D t} & +\frac{\rho}{r^{2}} \frac{\partial\left(v_{r} r^{2}\right)}{\partial r}=0, \\
\rho \frac{D Y_{i}}{D t} & =-\frac{1}{r^{2}} \frac{\partial}{\partial r}\left(r^{2} j_{i}^{\mathrm{diff}}\right), \\
\rho \frac{D v_{r}}{D t} & =-\frac{\partial p}{\partial r}+\frac{1}{r^{2}} \frac{\partial r^{2} \tau_{r r}}{\partial r}-\frac{\tau_{\theta \theta}+\tau_{\phi \phi}}{r}, \\
\rho \frac{D e}{D t} & =-\frac{p}{r^{2}} \frac{\partial\left(v_{r} r^{2}\right)}{\partial r}-\frac{1}{r^{2}} \frac{\partial\left(r^{2} q_{r}\right)}{\partial r}+\phi_{v},
\end{aligned}
$$

where $r$ is the radial coordinate, $Y_{i}$ is the mass fraction of the $i$ th component and $j_{i}^{\text {diff }}$ is the diffusive mass flux, $p$ is the pressure, $T$ is temperature, $\tau$ is the viscous stress tensor, $e$ is the specific internal energy, $q_{r}$ is the radial heat flux and $\phi_{v}$ is the viscous dissipation function. These equations apply for both the gas/vapor mixture inside the bubble and the liquid surrounding it.

The ideal gas equation is a good approximation of the real equation of state for both gas and vapor components inside the bubble in standard conditions. In the liquid, the definition of the sound speed in a pure liquid is typically used to relate pressure and density variations. Using the Fourier's and Fick's law to express the diffusive heat and mass flux and neglecting the enthalpy difference between the different components of the 
mixture at a given temperature we obtain the following set of equations that apply both, inside and outside the bubble,

$$
\begin{aligned}
& \frac{D \rho_{m}}{D t}+\frac{\rho_{m}}{r^{2}} \frac{\partial\left(v_{m} r^{2}\right)}{\partial r}=0 \\
& \rho_{m} \frac{D Y_{i, m}}{D t}=\frac{1}{r^{2}} \frac{\partial}{\partial r}\left(r^{2} D_{i / m}^{M} \frac{\partial Y_{i, m}}{\partial r}\right) \\
& \rho_{m} \frac{D v_{m}}{D t}=-\frac{\partial p_{m}}{\partial r} \\
& \rho_{m} c_{p, m} \frac{D T_{m}}{D t}=\alpha_{V} T_{m} \frac{D p_{m}}{D t}+\frac{1}{r^{2}} \frac{\partial}{\partial r}\left(r^{2} \kappa_{m} \frac{\partial T_{m}}{\partial r}\right)+\frac{4}{3} \mu_{m}\left(\frac{\partial v_{m}}{\partial r}-\frac{v_{m}}{r}\right)^{2}
\end{aligned}
$$

where $c_{p, m}$ is the average specific heat, $\alpha_{V}$ is the thermal dilatation coefficient which is $\alpha_{V}=1 / T_{m}$ for an ideal gas and approximately zero for liquids, $\kappa_{m}$ is the average conductivity and $D_{i / m}^{M}$ is the diffusion coefficient of the $i$ th component in the mixture. The subindex $m$ is used to denote the average mixture properties. When the equations are applied inside the bubble the subindex $m$ will be replaced by $b$, when applied into the liquid, we will use the subindex $l$.

The boundary conditions used to solve the system above can be found for example in Hauke et al. (2007). At the bubble center, spherical symmetry imposes the radial gradients to be zero, (i.e. $\frac{\partial Y_{i, b}}{\partial r}=\frac{\partial \rho_{b}}{\partial r}=\frac{\partial T_{b}}{\partial r}=0$ ). The bubble velocity is also set to zero, $v_{b}(r=0, t)=0$.

Far from the bubble, pressure, temperature and all the species concentrations in the liquid are assumed to be known $\left(p_{l, \infty}, T_{0}, Y_{i, l, 0}\right)$.

The local balances at the infinitely thin interface relate the liquid and gas properties at both sides of the interface. The mass balance across the bubble is

$$
J=\rho_{g}\left(\dot{R}-v_{b}^{I}\right)=\rho_{l}\left(\dot{R}-v_{l}^{I}\right) \quad \text { at } r=R,
$$

where $J$ is the total evaporation mass flux across the interface, $R$ is the bubble radius, $\dot{R}$ is the interface velocity, and $v_{b}^{I}$ and $v_{l}^{I}$ denote respectively the fluid interface velocity in the bubble and in the liquid. The momentum balance accounting for mass transfer effects reads

$$
J\left(v_{b}^{I}-v_{l}^{I}\right)=p_{l}^{I}-p_{b}^{I}+\tau_{r r, l}-\tau_{r r, b}+\frac{2 \sigma}{R} \quad \text { at } r=R
$$

where $\sigma$ is the surface tension coefficient.

The total flux of the $i$ th component across the interface is calculated as the sum of the advective flux and the diffusive flux. As both fluxes must be equal at $r=R$, we obtain an equation for the conservation of the $i$ th component across the interface as

$$
-J Y_{i, l}-\rho_{l} D_{i / l}^{M} \frac{\partial Y_{i, l}}{\partial r}=-J Y_{i, b}-\rho_{g} D_{i / b}^{M} \frac{\partial Y_{i, b}}{\partial r} \quad \text { at } r=R .
$$

The energy balance at the interface establishes the relation among the energy fluxes in the liquid/gas boundaries. This equation can be approximated by using the total latent 
heat of evaporation, $\Delta H_{\mathrm{vap}}$, as

$$
\kappa_{l} \frac{\partial T_{l}}{\partial r}=\kappa_{g} \frac{\partial T_{g}}{\partial r}+\sum_{i=1}^{N} J_{i} \Delta H_{\text {vap }, \mathrm{i}} \quad \text { at } r=R,
$$

where $J_{i}$ is the flux across the interface of the $i t h$ component and $\Delta H_{\mathrm{vap}, \mathrm{i}}$ is the enthalpy of vaporization related to the phase change. Finally, we assume a continuous temperature profile across the interface so that the interface temperature is equal for both phases $\left(T_{g}(r=R)=T_{l}(r=R)=T_{\text {int }}\right)$.

To evaluate the flux of every component across the interface $J_{i}$ that finally determines the total flux $J=\sum_{i=1}^{N} J_{i}$ we can either impose equilibrium conditions at the interface at every instant, or assume that the flux is proportional to the difference between the equilibrium state and the current state. In fact, using the kinetic theory of gases, it is possible to obtain the total flux of vapor across the interface using the Hertz-KnudsenLangmuir expression (Knudsen 1915; Hertz 1982),

$$
J_{\text {vap }}=\alpha_{\text {evap }} \frac{\left(p_{\text {eq }}^{I}-p_{b, \text { vap }}^{I}\right)}{\sqrt{2 \pi r_{\text {vap }} T_{\text {int }}}}
$$

where $\alpha_{\text {evap }}$ is the accommodation coefficient, which is a measure of the ratio of the molecules hitting the interface that change the phase, $p_{b \text {,vap }}^{I}$ is the partial pressure of vapor at the interface, $r_{\text {vap }}$ is the vapor's perfect gas constant and $p_{\text {eq }}^{I}$ is the equilibrium pressure at the interface conditions. For the vapor, the equilibrium pressure can be obtained from the Clausius-Clapeyron relation,

$$
\frac{d p_{\mathrm{eq}}}{d T}=\Delta H_{\mathrm{vap}} \frac{p_{\mathrm{eq}}}{r_{\mathrm{vap}} T^{2}} .
$$

It is interesting to remark that the system of equations above simplifies in cases where the interface is assumed to be at equilibrium at every instant. As we will prove along the theoretical development, for low enough frequencies, transient mass transfer effects (Eq. 2.16) are not relevant. In this situation the total flux is given by diffusion assuming equilibrium conditions at the interface at every instant. Analogously, the model could be easily extended for soluble gases by using the equilibrium pressure (or concentration) given by the Henry's law. It must be noted that in this work we will not consider the presence of soluble gases in the liquid given that the mass and heat flux related to phase change of soluble components is usually negligible compared with that of vapor.

\section{Linearized solutions}

The set of equations described above can be simplified assuming that all variables oscillate around an equilibrium state with a given frequency $\omega$. Thus, for a given variable $y$, we look for solutions of the form $y=y_{0}\left(1+\Delta y e^{\imath \omega t}\right)$, where $\Delta y$ is a complex quantity to be determined. For the linear solution to be valid, $\Delta y \ll 1$.

At the local scale, the momentum and continuity equations in the liquid can be rewritten as the Rayleigh-Plesset equation, which relates pressure (or potential) variations induced by the far field with the local response of the bubble. Neglecting compressibility effects the modified Rayleigh-Plesset equation accounting for mass transfer effects reads 
(Prosperetti 1982)

$$
R \ddot{R}-\frac{R \dot{J}}{\rho_{l}}+\frac{3}{2}\left(\dot{R}-\frac{J}{\rho_{l}}\right)^{2}-2 \frac{J}{\rho_{l}}\left(\dot{R}-\frac{J}{\rho_{l}}\right)=\frac{p_{l}^{I}-p_{l, \infty}}{\rho_{l}},
$$

where $p_{l}^{I}$ can be expressed in terms of the properties inside the bubble using Eq. 2.13. In the following, we will consider the system of a bubble containing an inmiscible gas and a vapor with an equilibrium concentration $Y_{0}$ in a monocomponent liquid. This is representative of most of the gas/liquid systems as the most important flux across the interface is expected to be given by liquid vaporization. In the linear limit the flux across the interface is $J=J_{0} \Delta R e^{\imath \omega t}$, where $J_{0}$ is a complex quantity to be determined. Neglecting non-linear terms we simplify the modified Rayleigh-Plesset equation as

$$
-R_{0}^{2} \omega^{2} \Delta R-\frac{R_{0} J_{0} \imath \omega}{\rho_{l}} \Delta R=\frac{\Delta R}{\rho_{l}}\left(-p_{b, 0} \Phi+\frac{2 \sigma}{R_{0}}-4 \mu_{l} \imath \omega\right)-\frac{p_{l, 0} \Delta p_{\infty}}{\rho_{l}},
$$

where $\Phi$ is a complex function to be obtained that is typically named transfer function and it serves to relate bubble radius oscillations and internal bubble pressure variations, such that $\Delta p_{b}=-\Phi \Delta R$.

The equation above can be rearranged to express the bubble radius variations in terms of the far field pressure variations as

$$
\Delta R=-\frac{1}{\omega_{0}^{2}-\omega^{2}+2 i \delta \omega} \Delta p_{\infty} \frac{p_{l, 0}}{\rho_{l} R_{0}^{2}},
$$

where

$$
\begin{array}{r}
\delta=\frac{2 \mu_{l}}{\rho_{l} R_{0}^{2}}+\frac{p_{g, 0}}{2 \omega \rho_{l} R_{0}^{2}} \Im(\Phi)+\frac{\operatorname{Re}\left(J_{0}\right)}{2 \rho_{l} R_{0}}, \\
\omega_{0}^{2}=\frac{p_{g 0}}{\rho_{l} R_{0}^{2}}\left(\operatorname{Re}(\Phi)-\frac{2 \sigma}{p_{g, 0} R_{0}}\right)+\frac{\omega \Im\left(J_{0}\right)}{\rho_{l} R_{0}}
\end{array}
$$

Eqs. 3.4 and 3.5 require the evaluation of the transfer function $\Phi$ and the flux across the interface $J_{0}$. Both variables can be obtained from the solution of the conservation equations inside the bubble. Following a development similar to that of Commander \& Prosperetti (1989), we consider a perfect gas and uniform pressure inside the bubble. The continuity equation inside the bubble can be rewritten using the energy equation as

$$
\frac{\dot{p}_{b}}{\gamma p_{b}}+\nabla \cdot\left(v_{b}-\frac{\gamma-1}{\gamma p_{b}} \kappa_{b} \nabla T_{b}\right)=0
$$

Integrating the equation from the bubble center to a given distance $r$, the radial velocity inside the bubble is given by

$$
v_{b}(r)=\frac{1}{\gamma p}\left((\gamma-1) \kappa_{b} \frac{\partial T}{\partial r}-\frac{1}{3} \dot{p}_{b} r\right) .
$$

This equation is evaluated at the interface to find an expression for the bubble's internal pressure,

$$
\dot{p}_{b}=\left.\frac{3}{R}(\gamma-1) \kappa_{b} \frac{\partial T_{b}}{\partial r}\right|_{r=R}-\frac{3}{R} \gamma p_{b} v_{b}^{I} .
$$

Using Eq. 2.12 to express the gas/vapor velocity at the interface $\left(v_{b}^{I}\right)$ as a function of 
the total evaporation flux and the interface velocity and linearizing we obtain

$$
\Delta p_{b}=-\left.3(\gamma-1) \imath \mathrm{Pe}_{b}^{-1} \frac{\gamma}{\gamma-1} \frac{\partial \Delta T_{b}}{\partial \zeta}\right|_{\zeta=1}-3 \gamma \Delta R-3 \gamma \imath J_{0}^{*} \Delta R,
$$

where $\zeta=r / R$ is the nondimensional radial distance, $\mathrm{Pe}_{b}=\frac{\omega R_{0}^{2}}{D_{b}^{T}}$ is the bubble's Peclet number defined using the bubble's thermal diffusitivity $D_{b}^{T}$, and the nondimensional mass transfer flux is

$$
J_{0}^{*}=\frac{J_{0}}{\rho_{b, 0} R_{0} \omega} .
$$

Assuming that only one component (denoted with the subindex $w$ ) is vaporized and that the vapor pressure solely depends on temperature, we can evaluate the mass transfer flux from the linearization of Eq. 2.16 to obtain

$$
J_{0}^{*} \Delta R=J_{\max }^{*}\left[\frac{\partial p_{\mathrm{eq}}}{\partial T} \frac{T_{0}}{p_{\mathrm{eq}}} \Delta T_{b}(\zeta=1)-\Delta p_{b}+\Delta Y(\zeta=1)\right],
$$

where $J_{\max }^{*}$ is a nondimensional flux defined as

$$
J_{\max }^{*} \equiv \alpha_{\text {evap }} \frac{p_{\text {eq }, 0}}{\rho_{b, 0} R_{0} \omega \sqrt{2 \pi r_{w} T_{0}}} .
$$

Equation 3.11 requires to know the vapor mass fraction variations and the temperature variations at the interface. To obtain $\Delta Y(\zeta=1)$ we solve the linear transport equation for the vapor inside the bubble. The transport equation (Eq. 2.9) is written in a nondimensional form using the Sherwood number, $\mathrm{Sh}_{D}=\frac{\omega R_{0}^{2}}{D_{w / b}}$, where $D_{w / b}$ is the diffusion coefficient of the vapor inside the bubble mixture,

$$
\operatorname{Sh}_{D} \Delta Y+i \frac{1}{\zeta^{2}} \frac{\partial}{\partial \zeta}\left(\zeta^{2} \frac{\partial \Delta Y}{\partial \zeta}\right)=0
$$

Its general solution,

$$
\Delta Y(\zeta)=C_{D} \frac{\sinh \left(\sqrt{\operatorname{Sh}_{D} \imath} \zeta\right)}{\zeta}
$$

is particularized using the boundary condition for component $w$ at the interface (Eq. 2.14)

$$
-J_{0}^{*} \Delta R=-J_{0}^{*} Y_{0} \Delta R-\left.\operatorname{Sh}_{D}^{-1} Y_{0} \frac{\partial \Delta Y}{\partial \zeta}\right|_{\zeta=1},
$$

where we have imposed $Y_{w, l}=1$. Thus, we can determine the constant $C_{D}$ to write the evolution of the mass fraction at the interface as

$$
\Delta Y(\zeta=1)=J_{0}^{*} \frac{\mathrm{Sh}_{D}}{\sqrt{\imath \mathrm{Sh}_{D}} \operatorname{coth}\left(\sqrt{\imath \mathrm{Sh}_{D}}\right)-1} \frac{1-Y_{0}}{Y_{0}} \Delta R .
$$

The temperature variations at the interface are obtained using the energy equation inside the bubble (Eq. 2.11). The nondimensional linear version of this equation,

$$
\Delta T_{b}-\Delta p_{b} \frac{\gamma-1}{\gamma}+\imath \mathrm{Pe}_{b}^{-1} \frac{1}{\zeta^{2}} \frac{\partial}{\partial \zeta}\left(\zeta^{2} \frac{\partial \Delta T_{b}}{\partial \zeta}\right)=0
$$

has the following general solution,

$$
\Delta T_{b}(\zeta)=\Delta p_{b} \frac{\gamma-1}{\gamma}+C_{1} \frac{\exp \left(-\sqrt{\mathrm{Pe}_{b} \imath} \zeta\right)}{\zeta}+C_{2} \frac{\exp \left(\sqrt{\mathrm{Pe}_{b} \imath} \zeta\right)}{\zeta} .
$$


Because the temperature is finite at $\zeta=0$, we find that $C_{1}=-C_{2}=-C_{0} / 2$. Thus, the interface's temperature variation is given by

$$
\Delta T_{b}(\zeta=1)=\Delta p_{b} \frac{\gamma-1}{\gamma}+C_{0} \sinh \left(\sqrt{\mathrm{Pe}_{b} \imath}\right)
$$

Plugging Eqs. 3.16 and 3.19 into Eq. 3.11 we obtain the following explicit relation for the mass flux across the interface

$$
J_{0}^{*} \Delta R=J_{c}^{*}\left(\left(\Delta H_{\text {vap }}^{*}-1\right) \Delta p_{b}+\Delta H_{\text {vap }}^{*} \frac{\gamma}{\gamma-1} C_{0} \sinh \left(\sqrt{\mathrm{Pe}_{b} \imath}\right)\right),
$$

where we have defined a nondimensional enthalpy of vaporization as $\Delta H_{\mathrm{vap}}^{*}=\frac{\Delta H_{\mathrm{vap}}}{c_{p, b} T_{0}}$ and a characteristic nondimensional flux

$$
J_{c}^{*}=\frac{J_{\max }^{*}}{1+J_{\max }^{*} \frac{\operatorname{Sh}_{D}}{\sqrt{\imath \operatorname{Sh}_{D}} \operatorname{coth}\left(\sqrt{\imath \mathrm{Sh}_{D}}\right)-1} \frac{1-Y_{0}}{Y_{0}}} .
$$

Note that this quantity provides a quantification about the influence of transient mass transfer effects. In fact, we can write Eq. 3.21 using the characteristic nondimensional mass flux, $J_{c \text {,eq }}^{*}$ that is obtained by assuming that equilibrium conditions prevail at the interface at every instant (see appendix A for details about the derivation of $J_{c \text {,eq }}^{*}$ ),

$$
J_{c}^{*}=\frac{J_{\max }^{*}}{1+J_{\max }^{*}\left(J_{c, \text { eq }}^{*}\right)^{-1}} .
$$

When the characteristic mass flux related to transient effects is much faster than the one at equilibrium conditions $\left(J_{\max }^{*} \gg J_{c \text {,eq }}^{*}\right)$, the mass transfer is controlled by the vapor diffusion inside the bubble: $J_{c}^{*} \approx J_{c \text {, eq }}^{*}$. In the opposite limit $\left(J_{\max }^{*} \ll J_{c \text {,eq }}^{*}\right)$, transient mass transfer effects control the mass flux across the interface: $J_{c}^{*} \approx J_{\max }^{*}$.

To obtain the value of the integration constant $C_{0}$ in Eq. 3.20 one needs to solve the energy equation in the liquid (Eq. 2.11). In its linearized form this equation reads

$$
\Delta T_{l}=-\imath \mathrm{Pe}_{l}^{-1} \frac{1}{\zeta^{2}} \frac{\partial}{\partial \zeta}\left(\zeta^{2} \frac{\partial \Delta T_{l}}{\partial \zeta}\right)
$$

and its general solution is

$$
\Delta T_{l}(\zeta)=C_{3} \frac{\exp \left(-\sqrt{\mathrm{Pe}_{l} \imath} \zeta\right)}{\zeta}+C_{4} \frac{\exp \left(\sqrt{\mathrm{Pe}_{l} \imath} \zeta\right)}{\zeta}
$$

where $\mathrm{Pe}_{l}=\frac{\omega R_{0}^{2}}{D_{l}^{T}}$ is the liquid's Peclet number. Note that consistent with the derivation of the far field equations, the effect of viscous dissipation as well as thermal dilatation on the temperature variations have been neglected. The particular solution of this equation requires to evaluate the liquid's temperature at some distance $\zeta_{\infty}$. In the dilute limit, one can consider that the temperature far from the bubble is constant and equal to $T_{0}$. In this case we find $C_{4}=0$.

The value of the free constants $C_{3}$ and $C_{0}$ in Eqs. 3.19 and 3.24 are obtained applying the boundary conditions at the interface. First, we assume that the interface's temperature has to be continuous. Thus we write,

$$
C_{3} \exp \left(-\sqrt{\mathrm{Pe}_{l} \imath}\right)=\Delta p_{b} \frac{\gamma-1}{\gamma}+C_{0} \sinh \left(\sqrt{\mathrm{Pe}_{b} i}\right) .
$$


In addition, the energy balance across the interface (Eq. 2.15) establishes that

$$
\left.\frac{\partial \Delta T_{l}}{\partial \zeta}\right|_{\zeta=1}=\left.\frac{\kappa_{b}}{\kappa_{l}} \frac{\partial \Delta T_{b}}{\partial \zeta}\right|_{\zeta=1}+J_{0}^{*} \mathrm{Pe}_{b} \Delta H_{\mathrm{vap}}^{*} \Delta R
$$

Replacing the corresponding expressions for the temperature derivatives and using Eq. 3.25 to express $C_{3}$ as a function of $C_{0}$ we obtain

$$
C_{0}=-\frac{\frac{\gamma-1}{\gamma}\left(1-\Delta T_{c}^{I}\right)}{\sinh \left(\sqrt{\mathrm{Pe}_{b} i}\right)} \Delta p_{b}
$$

where the complex quantity $\Delta T_{c}^{I}$ is defined as

$$
\Delta T_{c}^{I}=\frac{\kappa_{b}}{\kappa_{l}} \frac{\sqrt{\mathrm{Pe}_{b} \imath} \operatorname{coth}\left(\sqrt{\mathrm{Pe}_{b} \imath}\right)-1+J_{c}^{*} \mathrm{Pe}_{b} \Delta H_{\text {vap }}^{*}\left(\frac{\gamma}{\gamma-1}\right)}{1+\sqrt{\mathrm{Pe}_{l} \imath}+\frac{\kappa_{b}}{\kappa_{l}}\left[\sqrt{\mathrm{Pe}_{b} \imath} \operatorname{coth}\left(\sqrt{\mathrm{Pe}_{b} \imath}\right)-1+J_{c}^{*} \mathrm{Pe}_{b}\left(\Delta H_{\text {vap }}^{*}\right)^{2} \frac{\gamma}{\gamma-1}\right]} .
$$

Upon substitution of the integration constant into Eq. 3.25 we can represent the temperature variations at the interface as

$$
\Delta T_{b}(\zeta=1)=\frac{\gamma-1}{\gamma} \Delta T_{c}^{I} \Delta p_{b}
$$

The temperature gradient inside the bubble evaluated at the interface is obtained from Eq. 3.19,

$$
\left.\frac{\partial \Delta T_{b}}{\partial \zeta}\right|_{\zeta=1}=-\frac{\gamma-1}{\gamma}\left(1-\Delta T_{c}^{I}\right)\left[\sqrt{\mathrm{Pe}_{b} \imath} \operatorname{coth}\left(\sqrt{\mathrm{Pe}_{b} \imath}\right)-1\right] \Delta p_{b},
$$

and the mass flux across the interface (Eq. 3.20) is expressed as a function of the bubble pressure variations as

$$
J_{0}^{*} \Delta R=J_{c}^{*}\left(\Delta H_{\mathrm{vap}}^{*} \Delta T_{c}^{I}-1\right) \Delta p_{b} .
$$

Replacing the expressions for the temperature gradient and mass flux (Eqs. 3.30 and 3.31) into Eq. 3.9 we finally obtain the transfer function $\Phi$ as

$$
\Phi=\frac{3 \gamma}{1-3(\gamma-1) \imath \mathrm{Pe}_{b}^{-1}\left[\sqrt{\mathrm{Pe}_{b} \imath} \operatorname{coth}\left(\sqrt{\mathrm{Pe}_{b} \imath}\right)-1\right]\left(1-\Delta T_{c}^{I}\right)+3 \gamma \imath J_{c}^{*}\left(\Delta H_{\mathrm{vap}}^{*} \Delta T_{c}^{I}-1\right)}
$$

This transfer function is a generalization of the classical expression for pure gas bubbles (Prosperetti et al. 1988) accounting for interface temperature variations and mass transfer effects across the interface. For instance, it is easy to check that we recover the classical solution for pure gas bubbles by imposing no net mass flux across the interface $\left(J_{c}^{*}=0\right)$ and by assuming that the interface temperature variations are zero $\left(\Delta T_{c}^{I}=0\right)$.

Figure 1 depicts the real and imaginary part of the transfer function obtained for an air/vapor bubble for various water vapor contents at constant atmospheric pressure. At large frequencies the bubbles tend to recover the adiabatic response irrespective of the vapor content. At low frequencies, the transfer function recovers the isothermal limit for pure gas bubbles. As the amount of vapor increases, the real part takes values below 1 at low frequencies. This value corresponds to the limiting case in which equilibrium conditions prevail inside the bubble at every instant. It can be proven that this result can also be found assuming that vapor and gas are both ideal gases at constant temperature (see appendix B). The imaginary part is also influenced by the vapor content. In general 

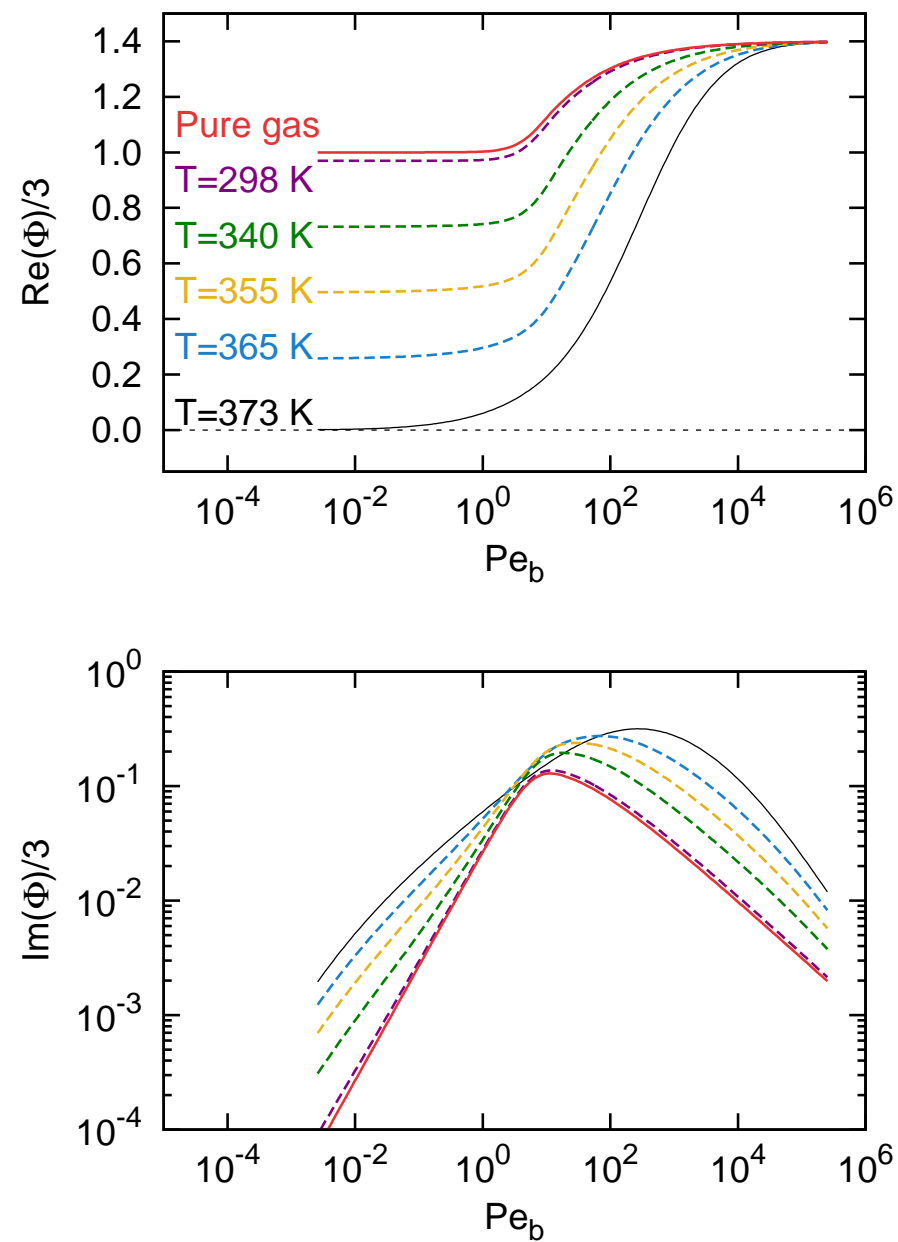

Figure 1: Color online. Influence of the vapor content on the real (top) and imaginary (bottom) part of the transfer function (Eq. 3.32) for various Peclet numbers. The values of the nondimensional quantities $J_{c}^{*}, \Delta H_{\mathrm{vap}}^{*}, \Delta T_{c}^{I}$ are obtained for a $100 \mu \mathrm{m}$ air/water vapor bubble at 1 atmosphere and varying temperature (from 25 to 100 degrees Celsius). The vapor content for the various curves are $Y_{0}=0,0.03,0.27,0.50,0.74,0.99$.

the imaginary part increases with the vapor content irrespective of the Peclet number although the influence of the vapor content on the imaginary part is especially notorious at low frequencies. Consistent with experimental observations, the influence of vaporization is completely negligible in gas/vapor bubbles at standard laboratory conditions (25 degree Celsius and 1 atmosphere) where the vapor void fraction is negligible compared with that of a gas.

Once we have obtained expressions for the transfer function (Eq. 3.32) and the mass flux across the interface (Eq. 3.31) we follow the classical procedure to linearize the averaged continuity and momentum equations (Eqs. 2.1 and 2.2) written as a wave equation 
of the form

$$
\frac{1}{c_{\mathrm{eff}}^{2}} \frac{\partial^{2} p_{l, \infty}}{\partial t^{2}}-\triangle p_{l, \infty}=4 \pi \rho \int_{0}^{\infty} a^{2} \ddot{R} f(a) d a,
$$

where $\ddot{R}=-\omega^{2} R_{0} \Delta R$ is the interface acceleration. Rewriting Eq. 3.33 as a wave equation for $p_{l, \infty}$, the complex sound speed, $c_{m}$, is given by

$$
\frac{1}{c_{m}^{2}}=\frac{1}{c^{2}}+4 \pi n \int_{0}^{\infty} \frac{a f(a) d a}{\omega_{0}^{2}-\omega^{2}+2 i \delta \omega}
$$

from which we obtain the phase velocity, $c_{p h}$, and attenuation, $Q^{-1}$, as

$$
\begin{array}{r}
c_{p h}=\left(\operatorname{Re}\left(\frac{1}{c_{m}}\right)\right)^{-1}, \\
Q^{-1}=-20 \log _{10}(e) \Im\left(\frac{\omega}{c_{m}}\right) .
\end{array}
$$

Using the expressions for the bubble resonant frequency and damping constant (Eqs. 3.4-3.5) we obtain the phase speed (Eq. 3.35) and attenuation (Eq. 3.36).

\section{Summary of equations}

As a summary of the full model equations for a bubbly liquid with vapor and an inmiscible gas, we solve for the averaged complex sound speed

$$
\frac{1}{c_{m}^{2}}=\frac{1}{c^{2}}+4 \pi n \int_{0}^{\infty} \frac{a f(a) d a}{\omega_{0}^{2}-\omega^{2}+2 i \delta \omega},
$$

in order to obtain the phase speed and attenuation as

$$
\begin{array}{r}
c_{p h}=\left(\operatorname{Re}\left(\frac{1}{c_{m}}\right)\right)^{-1}, \\
Q^{-1}=-20 \log _{10}(e) \Im\left(\frac{\omega}{c_{m}}\right) .
\end{array}
$$

The second term in Eq. 4.1 represents the influence of the bubble oscillation on the large scale wave propagation problem and it can be obtained in the linear regime by solving the conservation equations at the local scale (single bubble and its surrounding liquid). From the linearization of the Rayleigh-Plesset equation (e.g. continuity and momentum equation in the surrounding liquid) we find the following expressions for the bubble resonant frequency and the damping coefficient

$$
\begin{array}{r}
\delta=\frac{2 \mu_{l}}{\rho_{l} R_{0}^{2}}+\frac{p_{g, 0}}{2 \omega \rho_{l} R_{0}^{2}} \Im(\Phi)+\frac{1}{2} \operatorname{Re}\left(J_{0}^{*}\right) \frac{\rho_{b}}{\rho_{l}} \omega, \\
\omega_{0}^{2}=\frac{p_{g 0}}{\rho_{l} R_{0}^{2}}\left(\operatorname{Re}(\Phi)-\frac{2 \sigma}{p_{g, 0} R_{0}}\right)+\Im\left(J_{0}^{*}\right) \frac{\rho_{b}}{\rho_{l}} \omega^{2} .
\end{array}
$$

These properties require to evaluate the nondimensional mass transfer flux $J_{0}^{*}$ and the transfer function $\Phi$ that relates bubble's pressure and volume changes. From the solution of the conservation equations inside the bubble and the energy equation in the surrounding liquid we find the following expressions for these two quantities

$$
J_{0}^{*}=J_{c}^{*}\left(1-\Delta H_{\mathrm{vap}}^{*} \Delta T_{c}^{I}\right) \Phi,
$$


where $\mathrm{Pe}_{b}=\frac{\omega R_{0}^{2}}{D_{b}^{T}}, \Delta H_{\mathrm{vap}}^{*}=\frac{\Delta H_{\mathrm{vap}}}{c_{p, b} T_{0}}$, and $J_{c}^{*}$ and $\Delta T_{c}^{I}$ are the characteristic nondimensional flux and the nondimensional interface temperature variation. The general expression for the nondimensional flux $J_{c}^{*}$

$$
J_{c}^{*}=\frac{J_{\max }^{*}}{1+J_{\max }^{*}\left(J_{c, \text { eq }}^{*}\right)^{-1}},
$$

reveals that the total flux across the interface can be controlled either by vapor diffusion inside the bubble, $J_{c \text {,eq }}^{*}$, or by transient vaporization effects across the interface, $J_{\max }^{*}$. The diffusion flux

$$
J_{c, \text { eq }}^{*}=\frac{\sqrt{\imath \mathrm{Sh}_{D}} \operatorname{coth}\left(\sqrt{\imath \mathrm{Sh}_{D}}\right)-1}{\operatorname{Sh}_{D}} \frac{Y_{0}}{1-Y_{0}}
$$

depends on the Sherwood number $\mathrm{Sh}_{D}=\frac{\omega R_{0}^{2}}{D_{w / b}}$ and the amount of vapor $Y_{0}$. The characteristic transient mass flux is

$$
J_{\max }^{*}=\alpha_{\text {evap }} \frac{p_{\text {eq }, 0}}{\rho_{b, 0} R_{0} \omega \sqrt{2 \pi r_{w} T_{0}}} .
$$

Finally, the nondimensional interface temperature variation is a complex function depending on the heat and mass transfer processes taking place at the local scale and its general solution is

$$
\Delta T_{c}^{I}=\frac{\kappa_{b}}{\kappa_{l}} \frac{\sqrt{\mathrm{Pe}_{b} \imath} \operatorname{coth}\left(\sqrt{\mathrm{Pe}_{b} \imath}\right)-1+J_{c}^{*} \mathrm{Pe}_{b} \Delta H_{\mathrm{vap}}^{*}\left(\frac{\gamma}{\gamma-1}\right)}{1+\sqrt{\mathrm{Pe}_{l} \imath}+\frac{\kappa_{b}}{\kappa_{l}}\left[\sqrt{\mathrm{Pe}_{b} \imath} \operatorname{coth}\left(\sqrt{\mathrm{Pe}_{b} \imath}\right)-1+J_{c}^{*} \mathrm{Pe}_{b}\left(\Delta H_{\mathrm{vap}}^{*}\right)^{2} \frac{\gamma}{\gamma-1}\right]},
$$

where $\mathrm{Pe}_{l}=\frac{\omega R_{0}^{2}}{D_{l}^{T}}$

In the following sections we discuss the importance of mass transfer in the bubble dynamic response and the wave propagation properties of the effective medium as well as simplified solutions for limiting situations.

\section{The influence of mass transfer effects on the acoustic properties of the effective medium}

In this section we evaluate the importance of mass transfer effects in a system by comparing the results obtained with the full model with those provided by the classical linear theory that neglects mass transfer effects. We consider a mixture of monodisperse bubbles with average radius $100 \mu \mathrm{m}$ and bubble concentration of $\beta_{0}=10^{-4}$ ( $\mathrm{vol} / \mathrm{vol}$ ) measured at standard temperature (25 degrees Celsius) and pressure (1 atmosphere). We take the physical properties of an air/water system. Because the exact value of the accommodation coefficient is not clear yet (Gumerov et al. 2001) in this work we have taken $\alpha_{\text {evap }}=0.35$ (Yasui 1997; Hauke et al. 2007; Fuster et al. 2010), where we have neglected any influence of the temperature on this parameter.

Figure 2 contains the effective phase velocity and attenuation as a function of the vapor content and frequency obtained with the full model. The vapor content is controlled 
by varying the ambient temperature from 25 degrees Celsius to the normal boiling point of water (100 degrees Celsius) at constant ambient pressure. Under these conditions, the classical theory that neglects mass transfer effects does not predict any significant influence of the vapor content (or ambient temperature) on the results obtained. However, the results obtained with the full model reveal that mass transfer effects become relevant at low frequencies for bubbles with a large vapor content. At low frequencies the phase velocity is significantly reduced with respect to the value predicted when neglecting mass transfer effects whereas attenuation values increase various order of magnitude. The analysis of low frequency asymptotic solutions reached for low frequencies is postponed to the next section.

The differences in the formulation of the full model including mass transfer effects with respect to the classical formulation for pure gas bubbles can be condensed in two nondimensional quantities: $J_{0}^{*}$ as defined in Eq. 3.10, which is interpreted as the ratio between the interface velocity induced by mass transfer $\frac{J_{0}}{\rho_{b}} \Delta R$ and the actual interface velocity $R_{0} \omega \Delta R$, and $\Delta T_{c}^{I}$ as defined in Eq. 3.28, which is a measure of the nondimensional temperature variations at the interface (Eq. 3.29). When $J_{0}^{*}$ is close to one mass transfer controls of the evolution of the bubble radius, whereas when it is smaller than one the gas/vapor expansion controls the bubble radius evolution.

Figure 3 shows the modulus and the phase lag with respect to the bubble radius oscillations of the nondimensional mass transfer flux as a function of the Sherwood number and the vapor content (varied changing the ambient temperature from 25 to 100 degrees Celsius at 1 atmosphere). For a given vapor content, the evaporation flux reaches an asymptotic limit for low Sherwood numbers (low frequencies) which corresponds to the limit obtained assuming thermodynamic equilibrium inside the bubble at every instant (appendix B). This low frequency limit is also the maximum mass transfer flux across the interface for a given vapor content. Only for systems approaching saturation conditions the nondimensional mass transfer flux becomes of order one, meaning that evaporation and condensation processes take control of the dynamic response of the interface. On the contrary, for $\mathrm{Sh}_{D}>>1$ the bubble has almost no time to respond to pressure waves and the evaporation flux becomes negligible. In this high frequency regime neither phase speed nor attenuation are significantly influenced by mass transfer effects and we can conclude that the bubble dynamic response is governed by the gas/vapor expansion/compression.

The relevance of the interface temperature variations are captured by the nondimensional quantity $\Delta T_{c}^{I}$. Figure 4 shows $\Delta T_{c}^{I}$ as a function of the bubble's Peclet number, the nondimensional enthalpy of vaporization and the amount of vapor, which ultimately controls the mass flux across the interface. As expected, the interface temperature variations are negligible when the vapor content is low, irrespective of the forcing frequency and enthalpy of vaporization. In this case, the energy exchange due to evaporation/condensation is not significant. As we approach saturation conditions, the mass flux across the interface and the associated energy exchange make the interface temperature variations important. The sensitivity is maximal for a given enthalpy of vaporization that slightly depends on the forcing frequency. For reference, in the particular case of water the nondimensional enthalpy of vaporization, $\Delta H_{\text {vap }}^{*}$, takes values of order one, meaning that the interface temperature variations become important near saturation conditions. Regarding the frequency, the interface temperature variations are not important at low frequencies for both gas and vapor bubbles as the bubble has enough time to equilibrate its temper- 

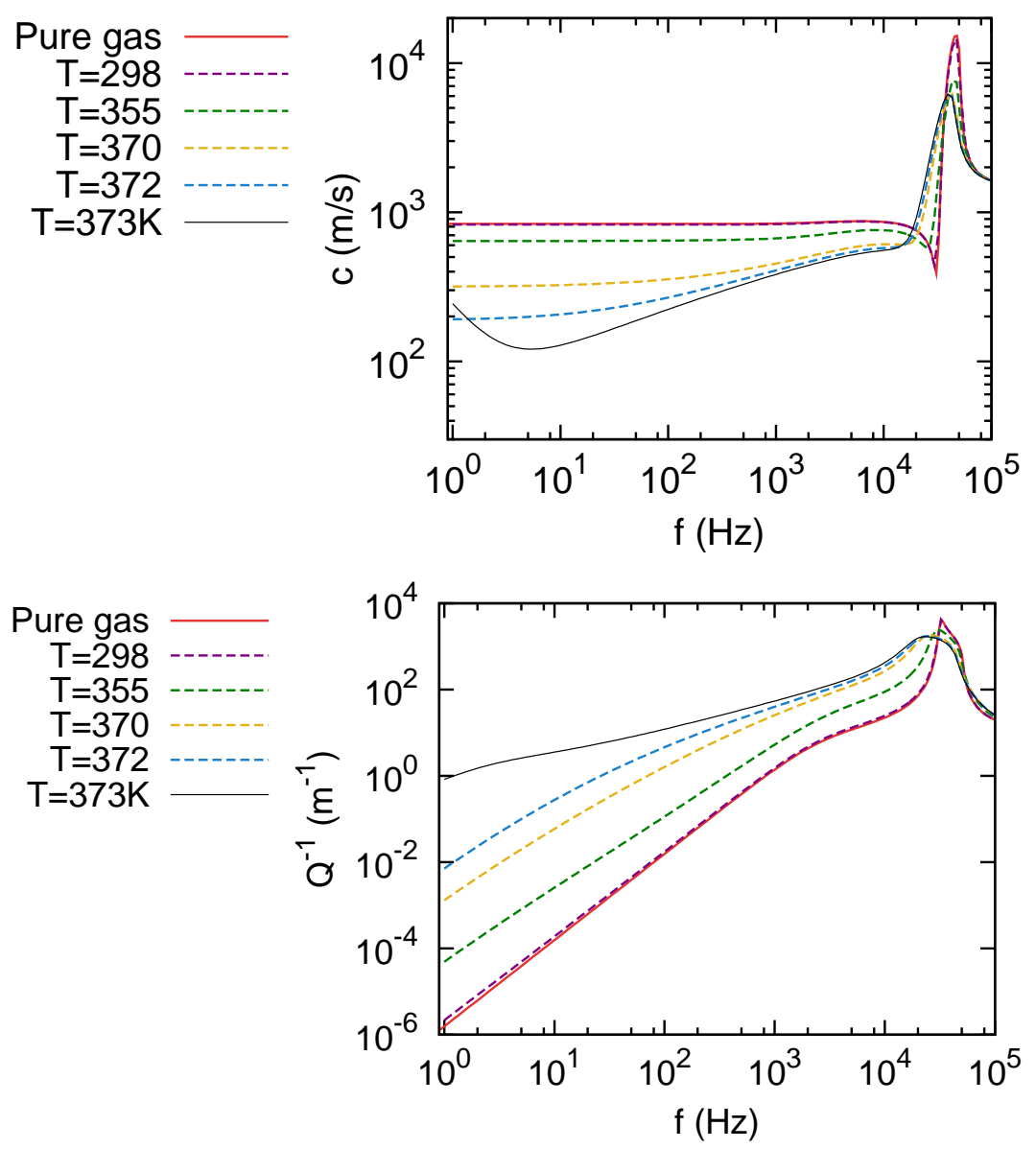

Figure 2: Color online. Influence of the vapor content on the phase velocity (top) and attenuation (bottom) curves obtained from the set of equations summarized in Section 4 for gas/vapor bubbles at $1 \mathrm{~atm}$ and various ambient temperatures. The vapor content for each condition is $Y_{0}=0.99,0.96,0.89,0.55,0.03$. For reference, the red line represents the solution of pure air bubbles $\left(Y_{0}=0\right)$ at 1 atm and 25 degrees Celsius. Mass transfer effects significantly modify both phase speed and attenuation curves for frequencies below resonance. In the low frequency limit, mass transfer decreases the phase velocity and increases attenuation.

ature with the surrounding liquid at every instant. As we increase the frequency (and therefore the Peclet number) the interface temperature variations are more important for bubbles with a low vapor content although in all cases the nondimensional values are close to zero. Thus, we can safely assume that the interface temperature is constant for gas bubbles in water irrespective of the forcing frequency. For bubbles with a large vapor content the situation is different because the interface temperature is influenced by the mass transfer flux. As this flux decreases when increasing the forcing frequency, there exists a range of intermediate frequencies for which the interface temperature variations become important in order to correctly predict the bubble radial oscillations. 

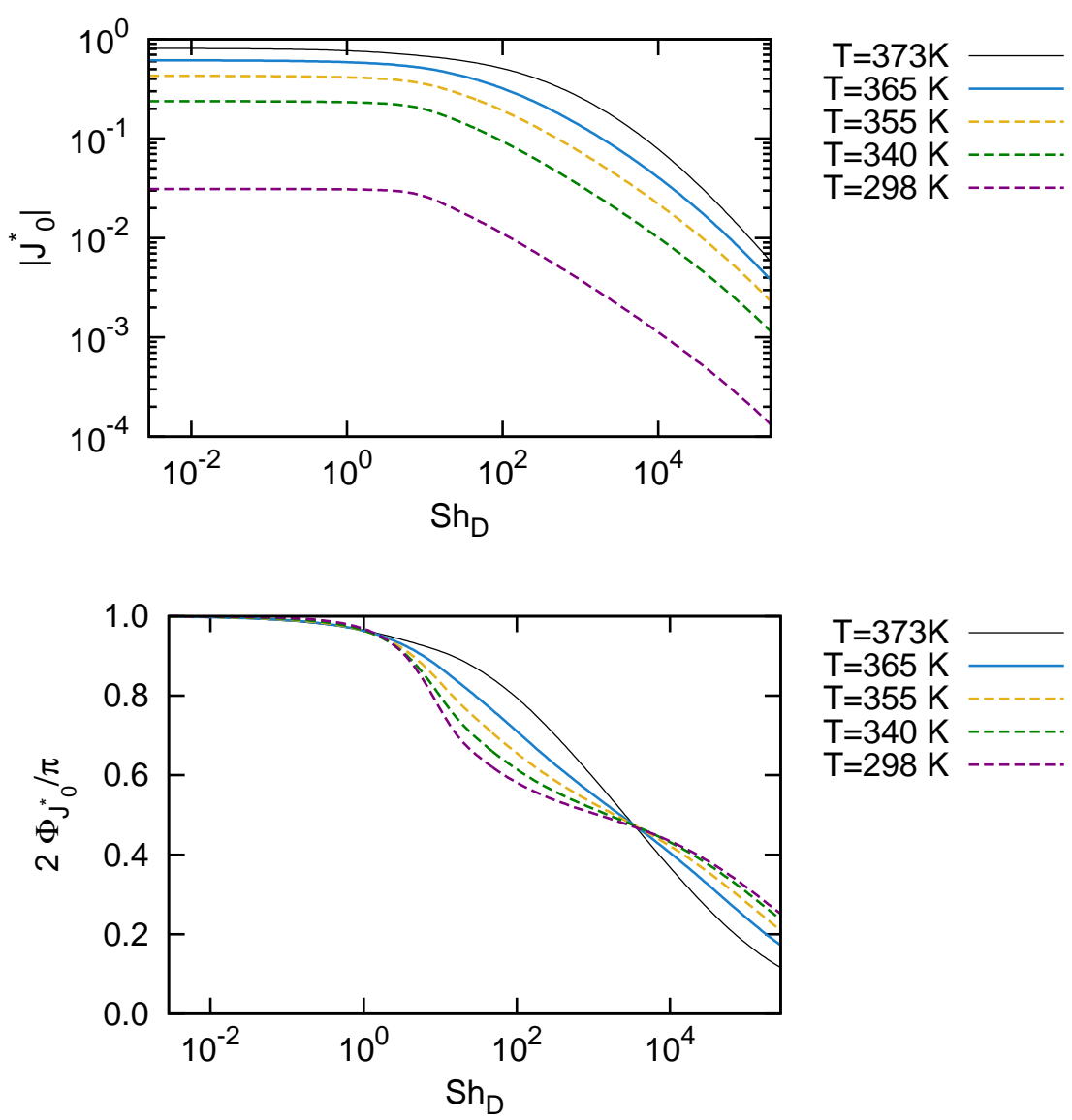

Figure 3: Color online. Top: Nondimensional evaporation flux (Eq. 4.6) as a function of the vapor content (varied by changing the ambient temperature at a constant pressure equal to $1 \mathrm{~atm}$ ) and the Sherwood number. Bottom: Phase lag between the bubble radius and flux oscillation as a function of the vapor content and the Sherwood number.

In order to gain further insight into the applicability of the constant interface temperature assumption Figure 5 compares the solution obtained with the full model with the solution obtained by impossing that the interface temperature remains constant $\left(\Delta T_{c}^{I}=0\right)$ still considering the mass transfer flux across the interface. The results are shown for a gas/vapor bubble at 25 degree Celsius (approximately $0.03 \%$ of vapor content) and 99.9 degree celsius $\left(Y_{0}=0.99\right)$. While at low ambient temperatures (low vapor content) the results obtained with $\Delta T_{c}^{I}=0$ accurately represent the solution obtained with the full model, we do observe significant differences when approaching saturation conditions. In this case the vaporization flux is significant and it is important to include interface temperature variations for an accurate estimation of the phase speed and attenuation at frequencies below the resonance frequency. Only if we decrease frequencies further down, the bubble response can be assumed to be isothermal and the phase velocities obtained assuming constant temperature accurately represent the solution of the full model. Note that this is not the case of the attenuation, where the interface temperature variations seem to have a significant influence on the predictions and must then be always consid- 

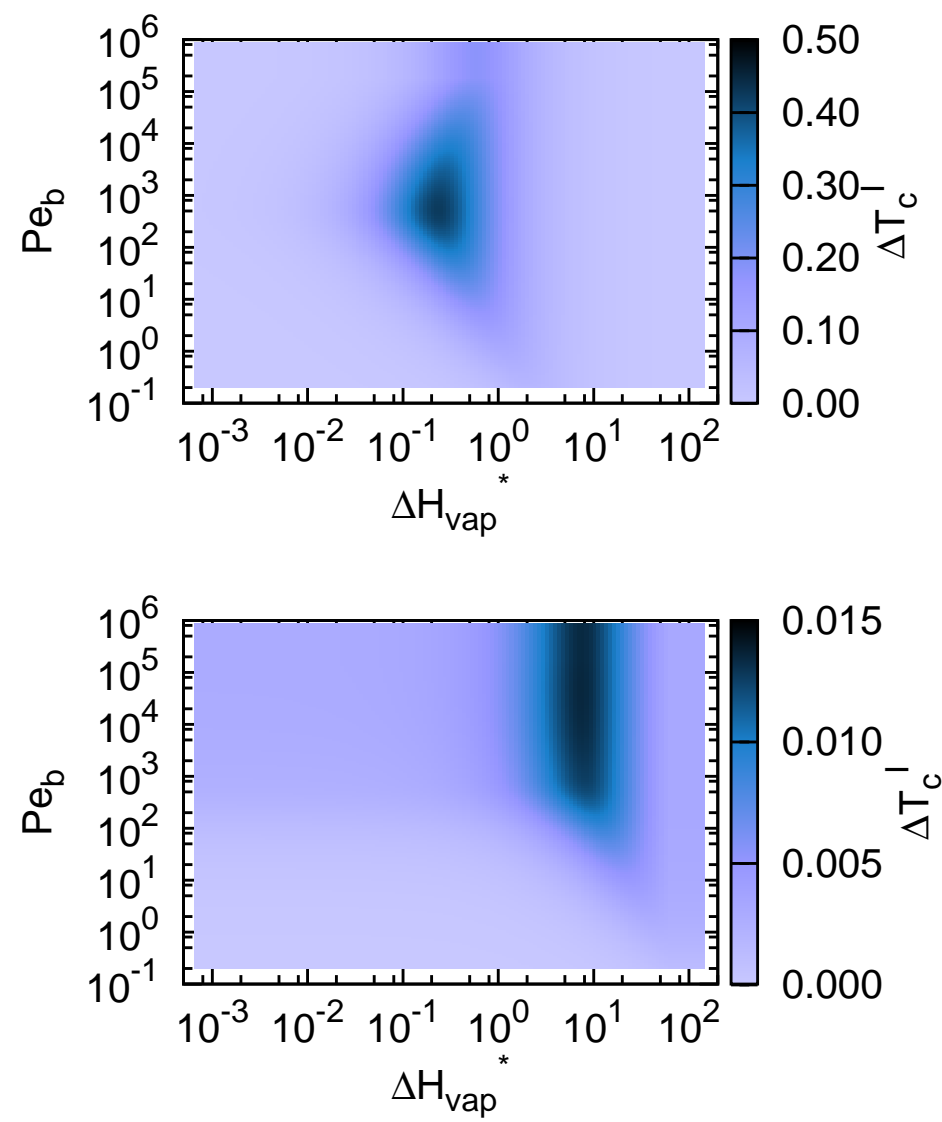

Figure 4: Color online. Dimensionless interface temperature variations (Eq. 4.11) as a function of the nondimensional enthalpy of vaporization, $\Delta H_{\text {vap }}^{*}$, and the bubble Peclet number, $\mathrm{Pe}_{b}$, for a $100 \mu \mathrm{m}$ bubble with large vapor content $Y_{0}=0.99$ (top) and low vapor content $Y_{0}=0.05$ (bottom).

ered in the predictions.

\section{Low frequency limits}

We have seen in the previous section that mass transfer effects are especially important for low frequency excitations. In this case both the bubble response and the process of wave propagation are significantly influenced by the mass and energy exchange across the interface. In order to gain further insight into the influence of mass transfer at low frequencies we derive limiting expressions for particular regimes. For frequencies well below the natural frequency and assuming monodisperse mixtures, it is readily shown that (Commander \& Prosperetti 1989)

$$
\frac{1}{c_{m}^{2}}=\frac{1}{c^{2}}+4 \pi \frac{n R_{0}}{\omega_{0}^{2}}\left(\frac{1-2 \imath \delta \omega}{\omega_{0}^{2}}\right) .
$$



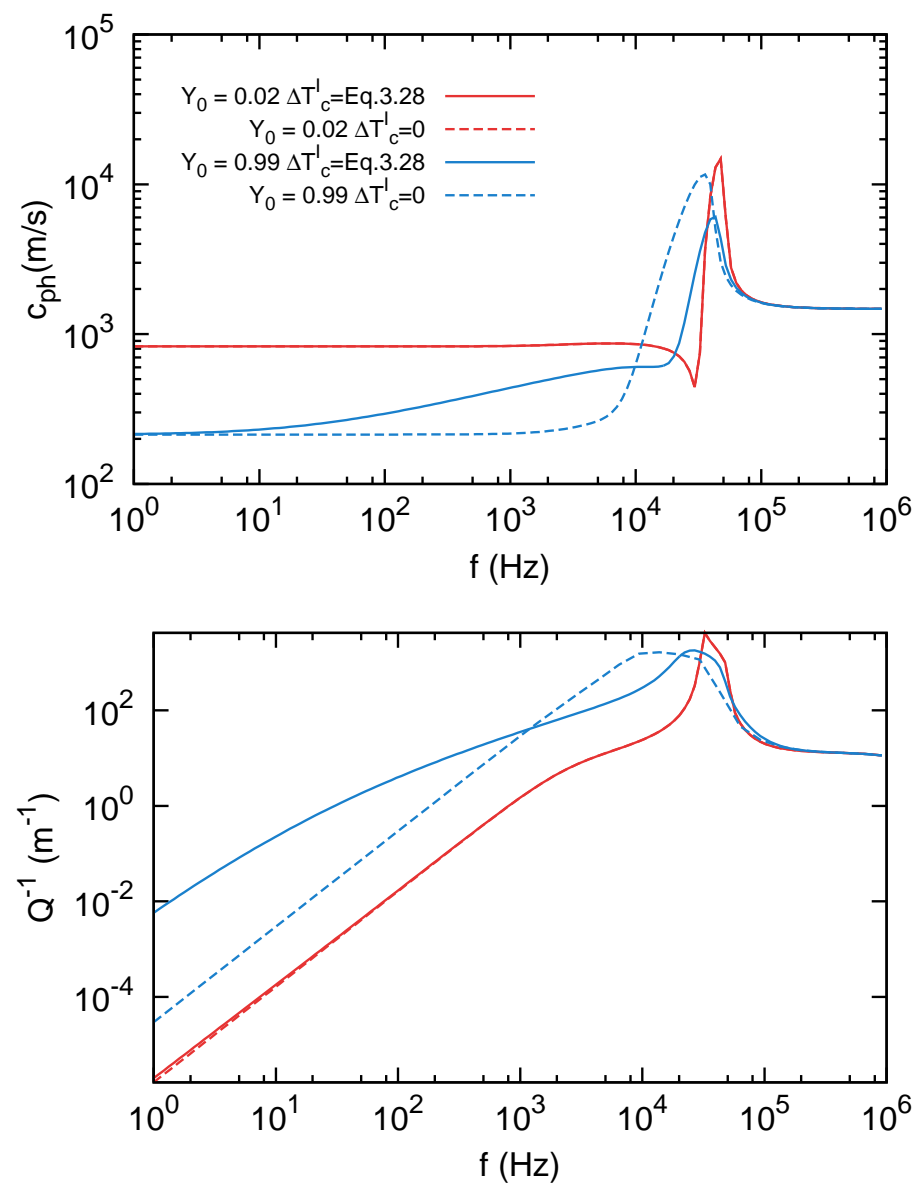

Figure 5: Color online. Phase speed (top) and attenuation curves (bottom) for an air/water system with $100 \mu \mathrm{m}$ bubbles with concentration $\beta_{0}=10^{-4}$ at $25 \mathrm{C}$ (red lines, $Y_{0}=0.03$ ) and $99.9 \mathrm{C}$ (blue lines, $Y_{0}=0.99$ ). The continuous lines represent the solution of the full model. The dashed lines represent the solution assuming a constant interface temperature. Both solutions overlap for systems containing gas bubbles whereas we observe significant differences between them for low frequency waves propagating in systems with bubbles with a large vapor content.

The limiting expressions for $\omega_{0}$ and $\delta$ depend on the evaporation flux and the transfer function. The nondimensional flux across the interface, given by Eq. 3.31, is $J_{0}^{*}=-J_{c}^{*} \Phi$. In the general case $J_{c}^{*}$ has to be obtained from Eq. 3.22, which in the low frequency limit tends to

$$
J_{c}^{*} \approx \frac{J_{\max }^{*}}{1-3 J_{\max }^{*} \imath \frac{1-Y_{0}}{Y_{0}}} \quad \text { when } \mathrm{Sh}_{\mathrm{D}}<1
$$

This expression can be further simplified when $J_{\max }^{*}>\frac{1}{3} \frac{Y_{0}}{1-Y_{0}}$, which can be written as a condition for the bubble's Peclet number,

$$
\mathrm{Pe}_{b}<\mathrm{Pe}_{c}^{I}=\frac{3 \alpha_{\text {evap }}}{\sqrt{2 \pi}}\left(1-Y_{0}\right) \frac{R_{0} \sqrt{r_{w} T}}{D_{b}^{T}} .
$$




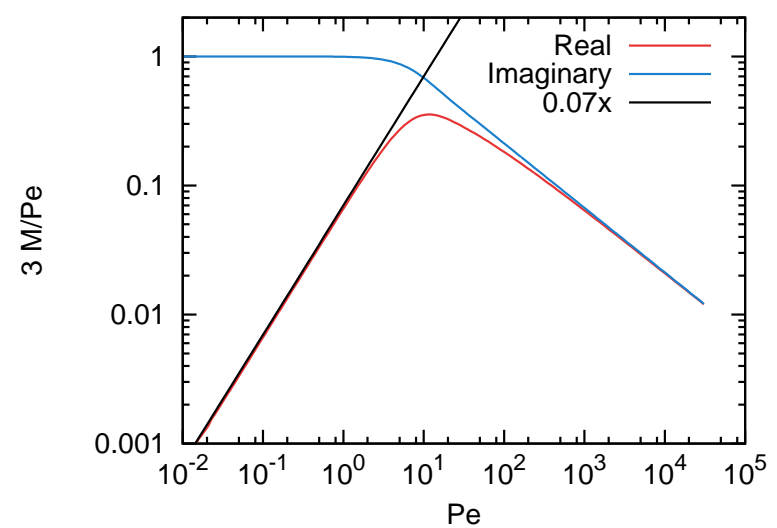

Figure 6: Color online. Real and imaginary parts of $3 \mathrm{Pe}^{-1} M$, where $M(\mathrm{Pe})=$ $\sqrt{\mathrm{Pe} \imath} \operatorname{coth}(\sqrt{\mathrm{Pe} \imath})-1$, as a function of Pe.

The right hand side $\left(\mathrm{Pe}_{c}^{I}\right)$ is identified with a critical Peclet number below which transient mass transfer effects are negligible. In this case equation 6.2 reduces to

$$
J_{c}^{*} \approx \frac{1}{3} \imath \frac{Y_{0}}{1-Y_{0}} \quad \text { when } \mathrm{Sh}_{\mathrm{D}}<1 \wedge \mathrm{Pe}_{\mathrm{b}}<\mathrm{Pe}_{\mathrm{c}}^{\mathrm{I}},
$$

which is equivalent to the low frequency solution obtained when assuming equilibrium conditions at the interface (see appendix A). Thus, we write the mass transfer flux across the interface and the transfer function as

$$
\begin{aligned}
& J_{0}^{*} \approx-\frac{1}{3} \imath \frac{Y_{0}}{1-Y_{0}} \Phi, \\
& \Phi \approx \frac{3\left(1-Y_{0}\right)}{1-\left(1-Y_{0}\right) \Delta T_{c}^{I} \frac{\gamma-1}{\gamma}-Y_{0} \Delta H_{\mathrm{vap}}^{*} \Delta T_{c}^{I}}
\end{aligned}
$$

which are valid when $\mathrm{Sh}_{D}<1 \wedge \mathrm{Pe}_{b}<\min \left(1, \mathrm{Pe}_{c}^{I}\right)$. Note that to derive the expression for the transfer function we have approximated the function $M(\mathrm{Pe})=\sqrt{\mathrm{Pe} \imath} \operatorname{coth}(\sqrt{\mathrm{Pe} \imath})-1$ by $M(\mathrm{Pe}) \approx \frac{\mathrm{Pe}}{3} \imath$, which is a reasonable assumption for $\mathrm{Pe}_{b}<1$ (Figure 6 ).

Both the mass transfer flux and the transfer function are a function of the interface temperature variations given by Eq. 3.28. For low frequencies, $\mathrm{Pe}_{b}<1$, Eq. 3.28 simplifies to

$$
\begin{array}{r}
\Delta T_{c}^{I} \approx \frac{\frac{\kappa_{b}}{\kappa_{l}} \frac{1}{3} \imath\left[1+\frac{Y_{0}}{1-Y_{0}} \Delta H_{\mathrm{vap}}^{*} \frac{\gamma}{\gamma-1}\right] \mathrm{Pe}_{b}}{1+\sqrt{\mathrm{Pe}_{l} \imath}+\frac{\kappa_{b}}{\kappa_{l}} \frac{1}{3} \imath\left[1+\frac{Y_{0}}{1-Y_{0}}\left(\Delta H_{\mathrm{vap}}^{*}\right)^{2} \frac{\gamma}{\gamma-1}\right] \mathrm{Pe}_{b}} \\
\text { when } \mathrm{Sh}_{\mathrm{D}}<1 \wedge \mathrm{Pe}_{\mathrm{b}}<\min \left(1, \mathrm{Pe}_{\mathrm{c}}^{\mathrm{I}}\right)
\end{array}
$$

When the interface temperature is isothermal, $\Delta T_{c}^{I}=0$, which is true for $\mathrm{Pe}_{b} \rightarrow 0$, the nondimensional mass flux across the interface and the transfer function for bubbles containing gas and vapor are

$$
\begin{array}{r}
J_{0}^{*}=Y_{0} \imath \\
\Phi \approx 3\left(1-Y_{0}\right) .
\end{array}
$$


Using these limiting expressions the final expressions for the phase speed and attenuation in the low frequency limit are given by

$$
\begin{aligned}
c_{\mathrm{ph}}^{2} & =\frac{c^{2}}{1+\frac{c^{2} \beta_{0} \rho_{l}}{\left(1-Y_{0}\right) p_{0}}}, \\
Q^{-1} & =20 \log _{10}(e) \frac{c \beta_{0} \delta \omega^{2} \rho_{l}^{2} R_{0}^{2}}{3 p_{0}^{2}\left(1-Y_{0}\right)^{2} \sqrt{1+\frac{c^{2} \beta_{0} \rho_{l}}{\left(1-Y_{0}\right) p_{0}}}} .
\end{aligned}
$$

For low void fractions $\left(\beta_{0}<\frac{p_{0}\left(1-Y_{0}\right)}{\rho_{l} c^{2}}\right)$, the phase speed tends to recover the liquid's sound speed. On the contrary, for large void fractions the phase speed is shown to be proportional to the gas content inside the bubble.

These limiting expressions are only expected to represent reasonably well the solution for dilute systems when $\mathrm{Sh}_{D}<1$ and $\mathrm{Pe}_{b}<\min \left(1, \mathrm{Pe}_{c}^{I}\right)$ and also when the interface temperature remains constant. This last condition introduces an additional constrain for $\mathrm{Pe}_{b}$ in order to reach the low frequency limits obtained above. From Eq. 6.7, we conclude that we can only assume constant interface temperature when

$$
\mathrm{Pe}_{b} \ll \mathrm{Pe}_{b, c}^{I I}=3 \frac{1-Y_{0}}{Y_{0}} \frac{\kappa_{l}}{\kappa_{b}} \frac{\gamma-1}{\gamma} \frac{1}{\left(\Delta H_{\text {vap }}^{*}\right)^{2}} .
$$

Thus, the frequency required to reach the low frequency limit is expected to be lower in systems with a low liquid thermal conductivity, a large content of vapor in the bubbles and a large enthalpy of vaporization. This effect can be clearly seen in Figure 7 where we compare the solution of systems with different non-dimensional values of the enthalpy of vaporization with the solution of a model where the interface temperature is assumed to be isothermal. As expected, all systems tend to the same low frequency limit for the phase speed, although we need to low the forcing frequency as the enthalpy of vaporization increases. In particular, in the case of the highest enthalpy of vaporization tested here (twice the enthalpy of vaporization of water) the curves tend to follow the low frequency limit for gas bubbles at frequencies between $10^{3}$ and $10^{4} \mathrm{~Hz}$. At these frequencies, the vaporization flux is limited by heat diffusion from the bulk towards the interface and the bubble dynamic response is mainly controlled by the expansion/compression rather than mass transfer effects. For lower frequencies, heat has enough time to diffuse and vaporization controls the effective compressibility of the medium. This remark is important if one wants to guarantee the validity of the low frequency limiting solutions in systems near saturation conditions, where it is not sufficient to satisfy that the excitation frequency is below the bubble's resonance frequency.

It is also interesting to compare the low frequency limit solutions obtained here with the classical solution for gas bubbles proposed by Wood (1930). Figure 8 compares the results obtained with the full model at $1 \mathrm{~Hz}$ as a function of the void fraction and the vapor content with the results obtained from Wood's theory. As it can be seen the phase speed is significantly lower for bubbles with a large vapor content than for pure gas bubbles. The effect of the vapor content can be also seen in Figure 9 where we see how both phase speed and attenuation converge to the solution for pure gas bubbles with vapor content tending to zero. For the phase velocity Wood's formula provides a good approximation of the exact solution only when the void fraction is sufficiently low. In the case of attenuation (Figure 9), $\delta$ strongly depends on the vapor content increasing 


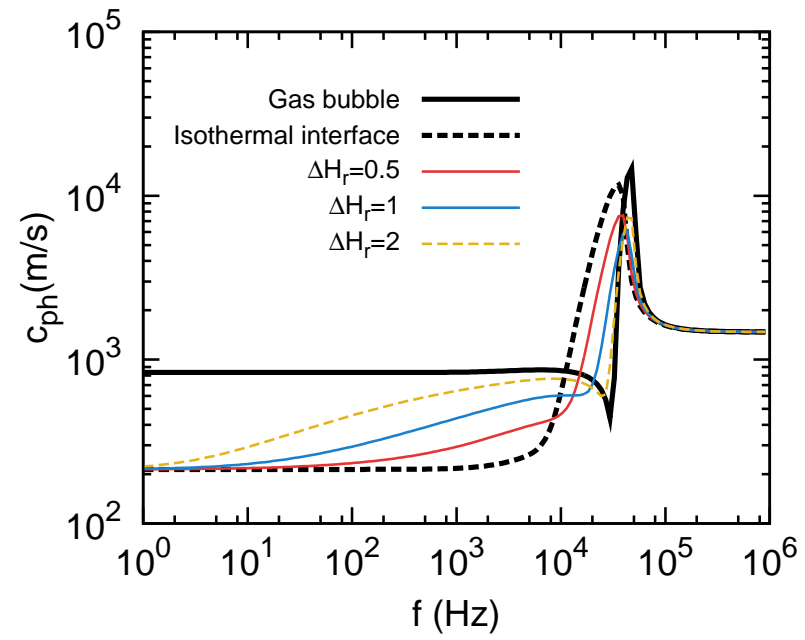

Figure 7: Color online. Phase speed predictions for systems with $100 \mu \mathrm{m}$ bubbles with concentration $\beta_{0}=10^{-4}$ at 1 atmosphere. The ambient temperature is fitted to obtain a vapor molar fraction of 0.95 . The dashed black line represents the predictions of the model accounting for mass transfer effects that imposes constant temperature at the interface. The solutions obtained from the full model are showed for different values of the enthalpy of vaporization which are given with respect to the enthalpy of vaporization of water, $\Delta H_{v a p}^{0}$, such that $\Delta H_{r}=\frac{\Delta H_{\text {vap }}}{\Delta H_{\text {vap }}^{0}}$. For reference, the continuous black line represents the predictions of the model for a pure gas bubble, which neglects mass transfer effects across the interface.

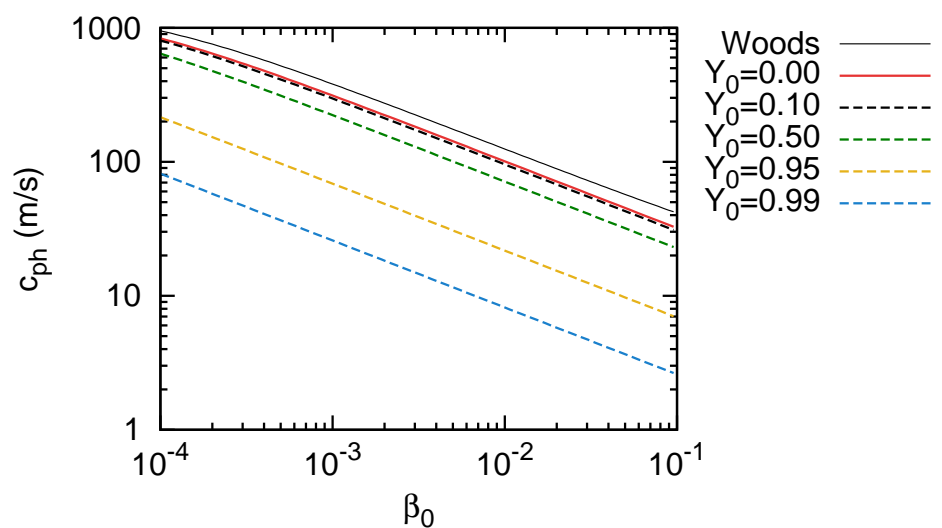

Figure 8: Color online. Phase speed in the low frequency limit $(f=1 \mathrm{~Hz})$ as a function of the void fraction and various vapor contents for a monodisperse bubble cloud of 100 $\mu \mathrm{m}$ bubbles. For reference the solution of pure gas bubbles and the solution of Wood (1930) is included. 

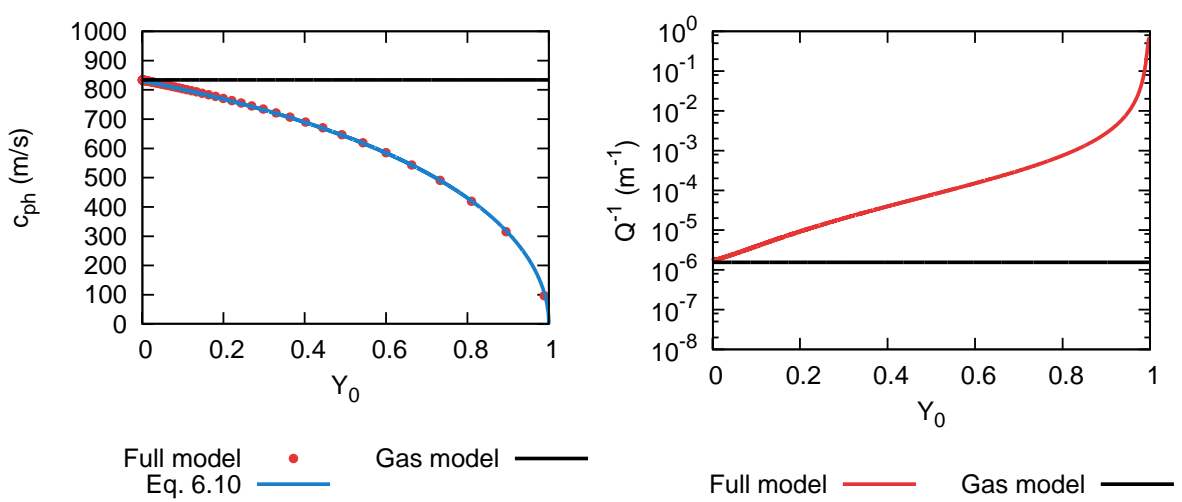

Figure 9: Color online. Phase speed (left) and attenuation (right) in the low frequency limit $(f=1 \mathrm{~Hz})$ as a function of the vapor content for a monodisperse bubble cloud of $100 \mu \mathrm{m}$ bubbles with concentration equal to $\beta_{0}=10^{-4}$. For reference we include the solution of pure gas bubbles and the approximate solution provided by Eqs. 6.10-6.11.

several orders of magnitude compared to the case of pure gas bubbles.

It is also worth mentioning that the limiting solutions of Eqs. 6.10-6.11 differ from the solutions obtained by Landau \& Lifshitz (1987) for pure liquid/vapor bubbly liquids, who derive an asymptotic solution considering that pressure and temperature at the large scale are coupled through the Clausius-Clapeyron equation. In this study we restrict ourselves to situations where the temperature far from the bubble remains constant, which is a reasonable assumption for dilute systems containing small amount of gas but it is not applicable for pure vapor bubbles and low frequencies where the heating/cooling of the bulk liquid may be relevant. Although the current model for pure vapor bubbles may be extended accounting for large scale thermal effects for the sake of simplicity we postpone the study of this situation for future investigation. In any case, it is important to note that the limiting solutions reported by Landau \& Lifshitz (1987) may be very difficult to obtain experimentally in linear regimes (Coste et al. 1990). On one side, very small amounts of gas make the vapor diffusion inside the bubble to be the controlling mechanism determining the vaporization flux. As we have seen above, the frequency threshold below which equilibrium conditions prevail inside the bubble at every instant tend to zero for vapor bubbles, which would make difficult to reach the low frequency limit in these situations. On the other side, for the particular case of pure vapor bubbles, the amplitude required to keep the linear regime valid tends to zero for low frequencies. This effect can be clearly seen in Figure 10, where we represent the effect of the vapor content on the sensitivity factor $S$ defined from Eq. 6.13 as

$$
S=\frac{\Delta R}{\Delta p_{\infty}}=-\frac{1}{\omega_{0}^{2}-\omega^{2}+2 i \delta \omega} \frac{p_{l, 0}}{\rho_{l} R_{0}^{2}} .
$$

This factor represents the nondimensional amplification factor of the bubble radius oscillation with respect to the external pressure excitation. Given that a pure vapor bubble is unable to reach equilibrium conditions when we perturb the pressure, the bubble tends to infinitely grow (or shrink) as we decrease the frequency. Small amounts of gas serve to kill this singular behavior, although the sensitivity of the bubble radius oscillation against the pressure pulse is still significantly influenced by the amount of vapor. 


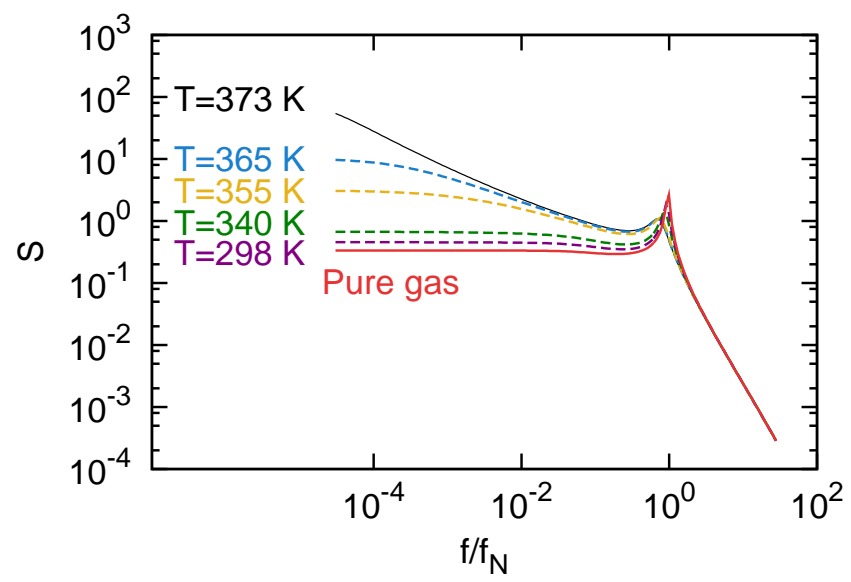

Figure 10: Color online. Sensitivity factor (as defined in Eq. 6.13) as a function of the vapor content and frequency for a $100 \mu \mathrm{m}$ gas/vapor bubble. The vapor content is changed by modifying the ambient temperature. As the amount of vapor increases, the bubble radial oscillation is more important for a constant nondimensional pressure wave amplitude.

\section{Conclusions}

This manuscript presents a generalization of the classical linear theory for dilute bubbly liquids that solves for the transient mass transfer effects across the interface as well as the heat transport in the liquid boundary layer. The proposed linear model converges to the solution of the classical linear theory for pure gas bubbles when the vapor content inside the vapor is negligible. Mass transfer effects are shown to play an important role in the phase speed and attenuation curves at frequencies below the bubble resonant frequency. At low frequencies the phase speed is significantly reduced while attenuation can increase various orders of magnitude with respect to the solution obtained when neglecting mass transfer effects.

Simplified solutions are derived for low frequency excitations. The conditions to be satisfied for these solutions to be valid are also obtained. We show that to reach the low frequency limit for the phase speed it is not sufficient to guarantee that the forcing frequency is lower than the bubble resonance frequency. For a general case, the Sherwood number, $\mathrm{Sh}_{D}=\frac{\omega R_{0}^{2}}{D_{w / b}}$, must be also smaller than one and the Peclet, $\mathrm{Pe}_{b}=\frac{\omega R_{0}^{2}}{D_{b}^{T}}$, must be smaller than the minimum among two characteristic Peclet numbers, $\mathrm{Pe}_{c}^{I}$ and $\mathrm{Pe}_{c}^{I I}$, in order to guarantee that thermodynamic equilibrium prevails at every instant. In systems with low liquid conductivity, large enthalpy of vaporization and large vapor content, the frequencies required to reach the isothermal limit for situations with a large vapor content can be extremely low. In this low frequency regime the bubble response is isothermal and it is possible to derive expressions that capture relatively well the influence of the vapor content on the acoustic properties of the effective medium. Thus, in systems close to saturation conditions and for frequencies below resonance, it is important to solve for the mass transfer flux across the bubble interface, the heat transport equation in the liquid and to account for the interface temperature variations in order to accurately 
obtain the phase speed and attenuation.

The authors would like to acknowledge Claudine Boehm and Philippe Berthet for their support and discussions.

\section{REFERENCES}

Ainslie, M.A. \& Leighton, T.G. 2011 Review of scattering and extinction cross-sections, damping factors, and resonance frequencies of a spherical gas bubble. J. Acoust. Soc. Am. 130 (5), 3184-3208.

Ando, K., Colonius, T. \& Brennen, C.E. 2009 Improvement of acoustic theory of ultrasonic waves in dilute bubbly liquids. J. Acoust. Soc. Am. 126, 69-74.

Ardron, KH \& Duffey, RB 1978 Acoustic wave propagation in a flowing liquid-vapour mixture. Int. J. Mult. Flow 4 (3), 303-322.

Chapman, R.B. \& Plesset, M.S. 1971 Thermal effects in the free oscillations of gas bubbles. J. Basic Eng. (Trans. ASME) 93, 373-376.

Cheyne, S.A., Stebbings, C.T. \& Roy, R.A. 1995 Phase velocity measurements in bubbly liquids using a fiber optic laser interferometer. J. Acoust. Soc. Am. 97 (3), 1621-1624.

Commander, K.W. \& Prosperetti, A. 1989 Linear pressure waves in bubbly liquids: Comparison between theory and experiments. J. Acoust. Soc. Am. 85, 732.

Coste, C, Laroche, C \& Fauve, S 1990 Sound propagation in a liquid with vapour bubbles. EPL (Europhys. Lett.) 11 (4), 343.

Fuster, D. \& Colonius, T. 2011 Modeling bubble clusters in compressible liquids. J. Fluid Mech. 688, 352-589.

Fuster, D., Conoir, J.M \& Colonius, T. 2014 Effect of direct bubble-bubble interactions on linear-wave propagation in bubbly liquids. Phys. Rev. E 90 (6), 063010.

Fuster, D., Hauke, G. \& Dopazo, C. 2010 Influence of accommodation coefficient on nonlinear bubble oscillations. J. Acoust. Soc. Am. 128, 5-10.

Gumerov, N. A., Hsiao, C.T. \& Goumilevski, A. G. 2001 Determination of the Accomodation Coefficient Using Vapor/Gas Bubble Dynamics in an Acoustic Field. Technical Report 1. California Institute of Technology, DYNAFLOW, Inc., Fulton, Maryland, see also URL http://gltrs.grc.nasa.gov/GLTRS (date last viewed 09/05).

Hao, Y. \& Prosperetti, A. 1999 The dynamics of vapor bubbles in acoustic pressure fields. Phys. Fluids 11 (8), 2008-2019.

Hauke, G., Fuster, D. \& Dopazo, C. 2007 Dynamics of a single cavitating and reacting bubble. Phys. Rev. E 75 (066310), 1-14.

HerTz, H. 1982 Über die Verdunstug der Flüssigkeiten, Inbesondere des Quecksilbers im lufteren Räume (on the evaporation of fluids, especially of mercury, in vacuum spaces). Ann. Phys. $17,177$.

Kieffer, S. W. 1977 Sound speed in liquid-gas mixtures: Water-air and water-steam. J. Geoph. Res. 82 (20), 2895-2904.

Knudsen, M. 1915 Maximum rate of vaporization of mercury. Ann. Phys. 47, 697.

Kuster, Guy T \& Toksöz, M NAfi 1974 Velocity and attenuation of seismic waves in twophase media: Part i. theoretical formulations. Geophysics 39 (5), 587-606.

Landau, L.D. \& Lifshitz, E.M. 1987 Fluid Mechanics. Pergamon Press.

Leroy, V., Strybulevych, A., Page, J.H. \& Scanlon, M.G. 2008 Sound velocity and attenuation in bubbly gels measured by transmission experiments. J. Acoust. Soc. Am. 123, 1931.

LyNnWORTH, L.C. 2013 Ultrasonic measurements for process control: theory, techniques, applications. Academic press.

Mecredy, RC \& Hamilton, LJ 1972 The effects of nonequilibrium heat, mass and momentum transfer on two-phase sound speed. Int. J. Heat and Mass Transf. 15 (1), 61-72.

Preston, A.T., Colonius, T. \& Brennen, C.E. 2007 A reduced order model of diffusive effects on the dynamics of bubbles. Phys. of Fluids 19 (123302), 1-19. 
Prosperetti, A. 1977 Thermal effects and damping mechanisms in the forced radial oscillations of gas bubbles in liquids. J. Acoust. Soc. Am. 61, 17-27.

Prosperetti, A. 1982 A generalization of the rayleigh-plesset equation of bubble dynamics. Phys. Fluids 25 (3), 409-410.

Prosperetti, A. 2015 The speed of sound in a gas-vapor bubbly liquid. Int. Focus 20140024.

Prosperetti, A., Crum, L.A. \& Commander, K.W. 1988 Nonlinear bubble dynamics. J. Acoust. Soc. Am. 83, 502-514.

Prosperetti, A. \& HaO, Y. 2002 Vapor bubbles in flow and acoustic fields. Ann. N. Y. Ac. Sci. 974 (1), 328-347.

SANGANI, ASHOK S 1991 A pairwise interaction theory for determining the linear acoustic properties of dilute bubbly liquids. J. Fluid Mech. 232, 221-284.

Saurel, R., Petitpas, F. \& Abgrall, R. 2008 Modelling phase transition in metastable liquids: application to cavitating and flashing flows. J. Fluid Mech. 607, 313-350.

Silberman, E. 1957 Sound velocity and attenuation in bubbly mixtures measured in standing wave tubes. J. Acoust. Soc. Am. 29, 8.

VAn WiJngaARDEn, L. 1968 On the equations of motion for mixtures of liquid and gas bubbles. J. Fluid Mech 33 (3), 465-474.

Wilson, P.S., Roy, R.A. \& Carey, W.M. 2005 Phase speed and attenuation in bubbly liquids inferred from impedance measurements near the individual bubble resonance frequency. $J$. Acoust. Soc. Am. 117 (4), 1895-1910.

Wood, A. B. 1930 A Textbook of Sound. London: G. Bell and Sons Ltd.

YAsui, K. 1997 Alternative model of single sonoluminiscence. Phys. Rev. E 56 (6), 6750-6760.

Zhang, DZ \& Prosperetti, A. 1997 Momentum and energy equations for disperse two-phase flows and their closure for dilute suspensions. Int. J. Mult. Flow 23, 425-453.

\section{Appendix A: Mass transfer flux assuming equilibrium conditions at the interface}

It is commonly assumed that equilibrium conditions prevail at the interface at every instant. Under these conditions, the partial water vapor pressure at the interface is given at every instant by the saturation pressure at the interface's temperature, $p_{\text {eq }}\left(T^{I}\right)=$ $p_{b} Y_{0} \frac{M_{b}}{M_{w}}$ whose linearized version is

$$
\Delta p_{\mathrm{eq}}^{I}=\Delta p_{b}+\Delta Y^{I}
$$

Replacing $\Delta p_{\text {eq }}^{I}$ by the expression given by the linear Clausious-Clapeyron relation (Eq. 2.17)

$$
\Delta p_{\mathrm{eq}}=\frac{\Delta H_{\mathrm{vap}}}{r_{w} T} \Delta T^{I}
$$

and using Eq. 3.16 to express the vapor fraction variations as a function of the mass flux across the interface, we can rewrite Eq. 8.1 as

$$
J_{0, \text { eq }}^{*} \Delta R=J_{c, \text { eq }}^{*}\left(\Delta H_{\text {vap }}^{*} \Delta T_{c}^{I}-1\right) \Delta p_{b}
$$

where

$$
J_{c, \text { eq }}^{*}=\left[\frac{\mathrm{Sh}_{D}}{\sqrt{\imath \mathrm{Sh}_{D}} \operatorname{coth}\left(\sqrt{\imath \mathrm{Sh}_{D}}\right)-1} \frac{1-Y_{0}}{Y_{0}}\right]^{-1} .
$$

For low frequencies $\left(\mathrm{Sh}_{D}<1\right)$, this equation simplifies to

$$
J_{c, \text { eq }}^{*} \approx \frac{1}{3} \imath \frac{Y_{0}}{1-Y_{0}} \quad \text { when } \mathrm{Sh}_{\mathrm{D}}<1 .
$$


9. Appendix B: Low frequency limit of the mass transfer flux assuming thermodynamic equilibrium inside the bubble

The limiting solution of the mass transfer flux for low frequencies can be also obtained by assuming that: (i) the temperature of the bubble remains constant at every instant and equal to the reference temperature, (ii) the vapor pressure inside the bubble is maintained at every instant. Thus, the water vapor mass can be obtained as

$$
m_{w}=\frac{p_{w} V}{r_{w} T}
$$

Because only the bubble volume changes, the flux is obtained as

$$
J=\frac{\dot{m}_{w}}{4 \pi R_{b}^{2}}=\frac{p_{w}}{r_{w} T} \dot{R}_{b}
$$

Linearizing the expression above we find

$$
J_{0} \Delta R=\frac{m_{w}}{V} \omega \imath R_{0} \Delta R,
$$

which in nondimensional form reads

$$
J_{0}^{*}=\frac{J_{0}}{R_{b} \rho_{b, 0} \omega}=Y_{0} \imath .
$$

